\title{
Global Exponential Tracking Control of a Mobile Robot System via a PE Condition
}

\author{
Warren E. Dixon, Darren M. Dawson, Senior Member, IEEE, Fumin Zhang, and Erkan Zergeroglu
}

\begin{abstract}
This paper presents the design of a differentiable, kinematic control law that achieves global asymptotic tracking. In addition, we also illustrate how the proposed kinematic controller provides global exponential tracking provided the reference trajectory satisfies a mild persistency of excitation (PE) condition. We also illustrate how the proposed kinematic controller can be slightly modified to provide for global asymptotic regulation of both the position and orientation of the mobile robot. Finally, we embed the differentiable kinematic controller inside of an adaptive controller that fosters global asymptotic tracking despite parametric uncertainty associated with the dynamic model. Experimental results are also provided to illustrate the performance of the proposed adaptive tracking controller.
\end{abstract}

Index Terms-Exponential tracking, mobile robot, nonholonomic, persistency of excitation, underactuated.

\section{INTRODUCTION}

$\mathbf{T}$ HE POSITION control problem of wheeled mobile robots (WMR's) has been a heavily researched area due to both the challenging theoretical nature of the problem (i.e., an underactuated nonlinear system under nonholonomic constraints) and its practical importance. In recent years, control researchers have targeted the problems of 1) tracking a time varying trajectory (which includes the path-following problem as a subset [7]); 2) regulating the mobile robot to a desired position/orientation; and 3) incorporating the effects of the dynamic model during the control design to enhance the overall performance and robustness of the closed-loop system. Researchers who have examined the above problems often cite that the regulation problem cannot be solved via a smooth, time-invariant state feedback law due to the implications of Brockett's condition [5]. In order to surmount this technical hurdle, researchers have proposed a variety of controllers to achieve setpoint regulation (see [21], [23], and the references therein for an in-depth review of the previous work) including: 1) discontinuous control laws; 2) piecewise continuous control laws; 3) smooth time-varying control laws; or 4) hybrid control laws. Specifically, in [4], Bloch et al. achieved local setpoint regulation for several different types of nonholonomic systems using a piecewise continuous control

Manuscript received June 1, 1999; revised October 31, 1999. This work was supported in part by the U.S. National Science Foundation Grants DMI-9457967, CMS-9634796, ECS-9619785, DMI-9813213, EPS-9630167, DOE Grant DE-FG07-96ER14728, Office of Naval Research Grant N00014-99-1-0589, and a DOC Grant. This paper was recommended by Associate Editor C. P. Neuman.

The authors are with the Department of Electrical and Computer Engineering, Clemson University, Clemson, SC 29634-0915 USA (e-mail ddawson@ces.clemson.edu).

Publisher Item Identifier S 1083-4419(00)01393-5. structure. Likewise, Canudas de Wit et al. [6] also constructed a piecewise smooth controller to exponentially regulate a WMR to a setpoint; unfortunately, due to the control structure, the orientation of the WMR is not arbitrary. In [23], Samson showcased several smooth, time-varying feedback controllers that could be utilized to asymptotically regulate a WMR to a desired setpoint. In addition to Samson's research, several smooth, time-varying controllers were also developed for other classes of nonholonomic systems in [8], [22], and [26]. Recently, Samson [24] provided a global asymptotic control solution for the setpoint regulation or the fixed reference-frame path following problem for a general class of nonholonomic systems. To enhance the transient performance Godhavn et al. [14] and McCloskey et al. [21] constructed control laws that locally $\rho$-exponentially (as well globally asymptotically) stabilized classes of nonholonomic systems. In addition to providing better transient performance, McCloskey et al. [21] also illustrated how the dynamic model of a WMR could be included during the control design under the assumption of exact model knowledge.

In addition to the setpoint regulation problem, several controllers have also been proposed for the reference robot tracking problem (i.e., the desired time-varying linear/angular velocity is specified). Specifically, in [18], Kanayama et al. obtained local asymptotic tracking using a continuous feedback control law for a linearized kinematic model. Using a continuous, linear control law for a linearized kinematic model similar to [18], Walsh et al. [26] obtained a local exponential stability result. Motivated by the desire to obtain global tracking (versus the aforementioned local results), Jiang et al. [16] developed a global asymptotic tracking controller; however, angular acceleration measurements were required. In [17], Jiang et al. provided semiglobal and global asymptotic tracking solutions for the general chained form system while eliminating the need for angular acceleration measurements that was required in [16]. In [13], Escobar et al. illustrated how the nonholonomic double integrator control problem (e.g., Heisenberg flywheel) can be exponentially stabilized by a redesigned field oriented induction motor controller; however, the controller exhibited singularities. Motivated by practical issues (i.e., parametric uncertainty in the dynamic model), Dong et al. [12] exploited the kinematic control structure proposed in [24] to construct a global adaptive asymptotic tracking control law for a class of nonholonomic systems. In [11], Dixon et al. presented a robust controller for the WMR that achieved global uniformly ultimately bounded tracking and regulation while rejecting parameter uncertainty and bounded disturbances in the dynamic model. We also note that several researchers (see [1], [7], and the references within) have proposed 
various controllers for the less stringent fixed reference-frame path following problem.

In this paper, we present a new, differentiable kinematic control law that achieves global asymptotic tracking 1 control. In addition, we also illustrate how the proposed kinematic controller provides for global exponential tracking provided the reference trajectory satisfies a mild ${ }^{2}$ persistency of excitation (PE) condition. Moreover, we illustrate how the proposed kinematic controller can be slightly modified to yield global asymptotic regulation of both the position and orientation of the mobile robot. Finally, we illustrate how the integrator backstepping approach can be used to embed the proposed differentiable kinematic controller inside of an adaptive controller that fosters global asymptotic tracking despite parametric uncertainty associated with the dynamic model (i.e., mass, inertia, and friction coefficients). From a retrospective view of literature, it seems evident that the proposed kinematic controller is novel in the respect that: 1) provided certain mild PE conditions on the reference trajectory are satisfied, a global exponential tracking result is obtained; 2) a global exponential tracking control scheme is crafted such that only minor modifications to the control structure are required to solve the global asymptotic regulation problem; and 3) to the best of our knowledge, this paper represents the first result that illustrates how the excitation of the reference trajectory can be used to improve the transient tracking performance.

The paper is organized as follows. In Section II, we present the kinematic model of the WMR and then transform the model into a form which facilitates the subsequent control development. In Section III, we present the kinematic control law, the closed-loop error system, and the corresponding stability analysis for the global asymptotic tracking controller. In Section IV, we develop the global exponential tracking result. In Section $\mathrm{V}$, we illustrate how simple modifications can be made to the proposed controller to obtain global asymptotic regulation. In Section VI, we develop the dynamic model for the WMR, formulate the adaptive dynamic control law, and then present the closed-loop error system and corresponding stability analysis for the global asymptotic tracking result. In Section VII, we illustrate the viability and effectiveness of the proposed adaptive tracking controller via experimental results. Concluding remarks are presented in Section VIII.

\section{KINEMATIC PROBLEM ForMULATION}

\section{A. WMR Kinematic Model}

The kinematic model for the so-called kinematic wheel under the nonholonomic constraint of pure rolling and nonslipping is given as follows [21]:

$$
\dot{q}=S(q) v
$$

where $q(t), \dot{q}(t) \in \Re^{3}$ are defined as

$$
q=\left[\begin{array}{lll}
x_{c} & y_{c} & \theta
\end{array}\right]^{T} \quad \dot{q}=\left[\begin{array}{lll}
\dot{x}_{c} & \dot{y}_{c} & \dot{\theta}
\end{array}\right]^{T}
$$

${ }^{1}$ The structure of the proposed kinematic controller is spawned from the induction motor controller presented in [10].

${ }^{2}$ The condition is mild in the sense that many reference trajectories satisfy the condition (e.g., a circle trajectory, sinusoidal trajectory, etc. can be exponentially tracked). $x_{c}(t), y_{c}(t)$, and $\theta(t) \in \Re^{1}$ denote the linear position and orientation, respectively, of the center of mass (COM) of the WMR, $\dot{x}_{c}(t), \dot{y}_{c}(t)$ denote the Cartesian components of the linear velocity of the COM, $\dot{\theta}(t) \in \Re^{1}$ denotes the angular velocity of the COM, the matrix $S(q) \in \Re^{3 \times 2}$ is defined as follows:

$$
S(q)=\left[\begin{array}{cc}
\cos \theta & 0 \\
\sin \theta & 0 \\
0 & 1
\end{array}\right]
$$

and the velocity vector $v(t) \in \Re^{2}$ is defined as

$$
v=\left[\begin{array}{ll}
v_{1} & v_{2}
\end{array}\right]^{T}=\left[\begin{array}{ll}
v_{l} & \dot{\theta}
\end{array}\right]^{T}
$$

with $v_{l}(t) \in \Re^{1}$ denoting the linear velocity of the COM of the WMR.

\section{B. Control Objective}

As defined in previous work (e.g., see [16] and [18]), the reference trajectory is generated via a reference robot which moves according to the following dynamic trajectory:

$$
\dot{q}_{r}=S\left(q_{r}\right) v_{r}
$$

where $S(\cdot)$ was defined in (3), $q_{r}(t)=\left[\begin{array}{lll}x_{r c}(t) & y_{r c}(t) & \theta_{r}(t)\end{array}\right]^{T} \in \Re^{3}$ is the desired time-varying position and orientation trajectory, and $v_{r}(t)=\left[\begin{array}{ll}v_{r 1}(t) & v_{r 2}(t)\end{array}\right]^{T} \in \Re^{2}$ is the reference time-varying linear and angular trajectory. With regard to (5), it is assumed that the signal $v_{r}(t)$ is constructed to produce the desired motion and that $v_{r}(t), \dot{v}_{r}(t), q_{r}(t)$, and $\dot{q}_{r}(t)$ are bounded for all time.

To facilitate the subsequent control synthesis and the corresponding stability proof, we define the following transformation:

$$
\begin{aligned}
{\left[\begin{array}{l}
w \\
z_{1} \\
z_{2}
\end{array}\right]=} & {\left[\begin{array}{ccc}
-\tilde{\theta} \cos \theta+2 \sin \theta & -\tilde{\theta} \sin \theta-2 \cos \theta & 0 \\
0 & 0 & 1 \\
\cos \theta & \sin \theta & 0
\end{array}\right] } \\
& \cdot\left[\begin{array}{c}
\tilde{x} \\
\tilde{y} \\
\tilde{\theta}
\end{array}\right]
\end{aligned}
$$

where $w(t) \in \Re^{1}$ and $z(t)=\left[z_{1}(t) \quad z_{2}(t)\right]^{T} \in \Re^{2}$ are auxiliary tracking error variables, $\tilde{x}(t), \tilde{y}(t), \tilde{\theta}(t) \in \Re^{1}$ are the difference between the actual Cartesian position and orientation of the COM and the desired position and orientation of the COM as follows:

$$
\tilde{x}=x_{c}-x_{r c} \quad \tilde{y}=y_{c}-y_{r c} \quad \tilde{\theta}=\theta-\theta_{r} .
$$

After taking the time derivative of (6), using (1)-(5), and (7), we can rewrite the tracking error dynamics in terms of the new variables defined in (6) as follows ${ }^{3}$

$$
\begin{aligned}
\dot{w} & =u^{T} J^{T} z+A z \\
\dot{z} & =u
\end{aligned}
$$

${ }^{3}$ Note that the structure of (8) is similar to the structure of the open-loop error system given in [10]. 
where $J \in \Re^{2 \times 2}$ is an auxiliary skew-symmetric matrix defined as

$$
J=\left[\begin{array}{rr}
0 & -1 \\
1 & 0
\end{array}\right]
$$

and the auxiliary row vector $A\left(z, v_{r}, t\right) \in \Re^{1 \times 2}$ is defined as

$$
A=\left[-2 v_{r 1} \frac{\sin \left(z_{1}\right)}{z_{1}} \quad 2 v_{r 2}\right] \text {. }
$$

The auxiliary variable $u(t)=\left[u_{1}(t) \quad u_{2}(t)\right]^{T} \in \Re^{2}$ utilized in (8) is used to simplify the transformed dynamics and is explicitly defined in terms of the WMR position and orientation, the WMR linear velocities, and the desired trajectory as follows:

$$
u=T^{-1} v-\left[\begin{array}{c}
v_{r 2} \\
v_{r 1} \cos \tilde{\theta}
\end{array}\right] \quad v=T u+\Pi
$$

where the auxiliary variables $T \in \Re^{2 \times 2}$ and $\Pi \in \Re^{2}$ are defined as follows:

$$
T=\left[\begin{array}{cc}
(\tilde{x} \sin \theta-\tilde{y} \cos \theta) & 1 \\
1 & 0
\end{array}\right]
$$

and

$$
\Pi=\left[\begin{array}{c}
v_{r 1} \cos \tilde{\theta}+v_{r 2}(\tilde{x} \sin \theta-\tilde{y} \cos \theta) \\
v_{r 2}
\end{array}\right] .
$$

\section{Kinematic Control DeVelopment}

Our control objective is to design a controller for the transformed WMR kinematic model given by (8). To facilitate the subsequent control development, we define an auxiliary error signal $\tilde{z}(t) \in \Re^{2}$ as the difference between the subsequently designed auxiliary signal $z_{d}(t) \in \Re^{2}$ and the transformed variable $z(t)$, defined in (6), as follows:

$$
\tilde{z}=z_{d}-z .
$$

\section{A. Control Formulation}

Based on the kinematic equations given in (8) and the subsequent stability analysis, we design the auxiliary signal $u(t)$ as follows:

$$
u=u_{a}-k_{3} z+u_{c}
$$

where the auxiliary control terms $u_{a}(t) \in \Re^{2}$ and $u_{c}(t) \in \Re^{2}$ are defined as

$$
u_{a}=k_{1} w J z_{d}+\Omega_{1} z_{d}
$$

and

$$
u_{c}=-\left(I_{2}+2 w J\right)^{-1}\left(2 w A^{T}\right)
$$

respectively, the auxiliary signal $z_{d}(t)$ is defined by the following dynamic oscillator-like relationship:

$$
\begin{aligned}
\dot{z}_{d} & =\left(k_{1}\left(w^{2}-z_{d}^{T} z_{d}\right)-k_{2}\right) z_{d}+J \Omega_{2} z_{d}+\frac{1}{2} u_{c} \\
\beta & =z_{d}^{T}(0) z_{d}(0)
\end{aligned}
$$

the auxiliary terms $\Omega_{1}(t) \in \Re^{1}$ and $\Omega_{2}(t) \in \Re^{1}$ are defined as

$$
\Omega_{1}=k_{1} w^{2}+k_{1}\left(w^{2}-z_{d}^{T} z_{d}\right)-k_{2}+k_{3}
$$

and

$$
\Omega_{2}=k_{1} w+w \Omega_{1}
$$

respectively, where $k_{1}, k_{2}, k_{3} \in \Re^{1}$ are positive, constant control gains, $I_{2}$ represents the standard $2 \times 2$ identity matrix, $\beta \in \Re^{1}$ is a positive constant, and $A\left(z, v_{r}, t\right)$ was defined in (10). Note that it is straightforward to show that the matrix $I_{2}+2 w J^{T}$ used in (17) is always invertible provided $w(t)$ remains bounded.

\section{B. Error System Development}

To facilitate the closed-loop error system development for $w(t)$, we substitute (15) for $u(t)$ defined in (8), add and subtract $u_{a}^{T} J z_{d}$ to the resulting expression, utilize (14), and exploit the skew symmetry of $J$ defined in (9) to rewrite the dynamics for $w(t)$ given by (8), as follows:

$$
\dot{w}=u_{a}^{T} J \tilde{z}-u_{a}^{T} J z_{d}+A z-u_{c}^{T} J z
$$

where the fact that $J^{T}=-J$ has been utilized. Finally, after substituting (16) for only the second occurrence of $u_{a}(t)$ in (21), substituting (17) for $u_{c}(t)$, utilizing the skew symmetry of $J$ defined in (9), and the facts that $J^{T} J=I_{2}$ and $J^{T}=-J$, we can obtain the final expression for the closed-loop error system as follows:

$$
\dot{w}=-k_{1} w z_{d}^{T} z_{d}+A z+2 w A\left(I_{2}+2 w J^{T}\right)^{-1} J z+u_{a}^{T} J \tilde{z} .
$$

To facilitate the subsequent stability analysis, we substitute (17) for $u_{c}(t)$ into (18) to yield the following form for the dynamics of $z_{d}(t)$

$$
\begin{aligned}
\dot{z}_{d}= & \left(k_{1}\left(w^{2}-z_{d}^{T} z_{d}\right)-k_{2}\right) z_{d} \\
& +J \Omega_{2} z_{d}-\left(I_{2}+2 w J\right)^{-1} w A^{T} .
\end{aligned}
$$

To determine the closed-loop error system for $\tilde{z}(t)$, we take the time derivative of (14), and then substitute (18) and (8) for $\dot{z}_{d}(t)$ and $\dot{z}(t)$, respectively, to obtain

$$
\dot{\tilde{z}}=\left(k_{1}\left(w^{2}-z_{d}^{T} z_{d}\right)-k_{2}\right) z_{d}+J \Omega_{2} z_{d}+\frac{1}{2} u_{c}-u .
$$

After substituting (15) into (24) for $u(t)$, and then substituting (16) for $u_{a}(t)$ in the resulting expression, we can rewrite the expression given by (24) as follows:

$$
\begin{aligned}
\dot{\tilde{z}}= & \left(k_{1}\left(w^{2}-z_{d}^{T} z_{d}\right)-k_{2}\right) z_{d}+J \Omega_{2} z_{d} \\
& -\frac{1}{2} u_{c}-k_{1} w J z_{d}-\Omega_{1} z_{d}+k_{3} z .
\end{aligned}
$$

After substituting (19) and (20) for $\Omega_{1}(t)$ and $\Omega_{2}(t)$ into (25), respectively, and then using the fact that $J J=-I_{2}$, we can cancel common terms and rearrange the resulting expression to obtain

$$
\dot{\tilde{z}}=-k_{3} \tilde{z}+w J\left[\Omega_{1} z_{d}+k_{1} w J z_{d}\right]-\frac{1}{2} u_{c}
$$


where (14) has been utilized. Finally, we substitute (17) for $u_{c}(t)$ to determine the final expression for the closed-loop error system as follows:

$$
\dot{\tilde{z}}=-k_{3} \tilde{z}+\left(I_{2}+2 w J\right)^{-1} w A^{T}+w J u_{a}
$$

where we have used the fact that the bracketed term in (26) is equal to $u_{a}(t)$ defined in (16).

\section{Stability Analysis}

Theorem 1: The kinematic controller given by (15)-(20) ensures global asymptotic tracking in the sense that

$$
\lim _{t \rightarrow \infty} \tilde{x}(t), \tilde{y}(t), \tilde{\theta}(t)=0
$$

provided the reference trajectory is selected such that

$$
\lim _{t \rightarrow \infty}\left\|v_{r}\right\| \neq 0
$$

Proof: To prove Theorem 1, we define the following nonnegative function denoted by $V\left(w, z_{d}, \tilde{z}, t\right) \in \Re^{1}$ as follows:

$$
V(t)=\frac{1}{2} w^{2}+\frac{1}{2} z_{d}^{T} z_{d}+\frac{1}{2} \tilde{z}^{T} \tilde{z} .
$$

After taking the time derivative of (30) and making the appropriate substitutions from (22), (23), and (27), we obtain the following expression:

$$
\begin{aligned}
\dot{V}= & w\left[u_{a}^{T} J \tilde{z}-k_{1} w z_{d}^{T} z_{d}+A z+2 w A\left(I_{2}+2 w J^{T}\right)^{-1} J z\right] \\
& +z_{d}^{T}\left[\left(k_{1}\left(w^{2}-z_{d}^{T} z_{d}\right)-k_{2}\right) z_{d}\right. \\
& \left.\quad+J \Omega_{2} z_{d}-\left(I_{2}+2 w J\right)^{-1} w A^{T}\right] \\
& +\tilde{z}^{T}\left[w J u_{a}-k_{3} \tilde{z}+\left(I_{2}+2 w J\right)^{-1} w A^{T}\right] .
\end{aligned}
$$

After utilizing the skew symmetry property of $J$ defined in (9), making use of the fact that $J^{T}=-J$, and cancelling common terms, we can upper bound (31) as follows:

$$
\begin{aligned}
\dot{V} \leq & -k_{2} z_{d}^{T} z_{d}-k_{3} \tilde{z}^{T} \tilde{z}+w A z \\
& +\left[w A\left(I_{2}+2 w J^{T}\right)^{-1}(2 w J)\right] z \\
& -\left[w A\left(I_{2}+2 w J^{T}\right)^{-1}\right] z
\end{aligned}
$$

where (14) has been utilized. Next, after utilizing the fact that $J^{T}=-J$, we can combine the bracketed terms in (32) as shown below

$$
\begin{aligned}
\dot{V} \leq & -k_{2} z_{d}^{T} z_{d}-k_{3} \tilde{z}^{T} \tilde{z}+w A z \\
& -w A\left[\left(I_{2}+2 w J^{T}\right)^{-1}\left(I_{2}+2 w J^{T}\right)\right] z .
\end{aligned}
$$

After noting that the bracketed term in (33) is equal to the identity matrix, we can cancel common terms to obtain the final upper bound for $\dot{V}(t)$ as follows:

$$
\dot{V} \leq-k_{2} z_{d}^{T} z_{d}-k_{3} \tilde{z}^{T} \tilde{z} .
$$

Based on (30) and (34), we can conclude that $V(t) \in \mathcal{L}_{\infty}$; thus, $w(t), z_{d}(t), \tilde{z}(t) \in \mathcal{L}_{\infty}$. Since $w(t), z_{d}(t), \tilde{z}(t) \in \mathcal{L}_{\infty}$, we can utilize (10), (14)-(20), (22), (27), and the fact that the reference trajectory is assumed to be bounded to conclude that $A(t), z(t), u(t), u_{a}(t), u_{c}(t), \Omega_{1}(t), \Omega_{2}(t), \dot{z}_{d}(t), \dot{w}(t), \dot{\tilde{z}}(t) \in$ $\mathcal{L}_{\infty}$. Since $\dot{z}_{d}(t), \dot{\tilde{z}}(t) \in \mathcal{L}_{\infty}$, we can utilize (14) to show that $\dot{z}(t) \in \mathcal{L}_{\infty}$ [since $\dot{w}(t), \dot{z}_{d}(t), \dot{\tilde{z}}(t), \dot{z}(t) \in \mathcal{L}_{\infty}$, we know that $w(t), z_{d}(t), \tilde{z}(t)$, and $z(t)$ are uniformly continuous]. In order to illustrate that the Cartesian position and orientation signals defined in (1) are bounded, we calculate the inverse transformation of (6) as follows:

$$
\left[\begin{array}{c}
\tilde{x} \\
\tilde{y} \\
\tilde{\theta}
\end{array}\right]=\left[\begin{array}{ccc}
0 & \frac{1}{2}(\tilde{\theta} \sin \theta+2 \cos \theta) & \frac{1}{2} \sin \theta \\
0 & -\frac{1}{2}(\tilde{\theta} \cos \theta-2 \sin \theta) & -\frac{1}{2} \cos \theta \\
1 & 0 & 0
\end{array}\right]\left[\begin{array}{c}
z_{1} \\
z_{2} \\
w
\end{array}\right] .
$$

Since $z(t) \in \mathcal{L}_{\infty}$, it is clear from (7) and (35) that $\tilde{\theta}(t), \theta(t)$ $\in \mathcal{L}_{\infty}$. Furthermore, from (7), (35), and the fact that $w(t), z(t)$, $\tilde{\theta}(t) \in \mathcal{L}_{\infty}$, we can conclude that $\tilde{x}(t), \tilde{y}(t), x_{c}(t), y_{c}(t) \in \mathcal{L}_{\infty}$. We can utilize (11), the assumption that the desired trajectory is bounded, and the fact that $\theta(t), u(t), \tilde{x}(t), \tilde{y}(t) \in \mathcal{L}_{\infty}$, to show that $v(t) \in \mathcal{L}_{\infty}$; therefore, it follows from (1)-(4) that $\dot{\theta}(t)$, $\dot{x}_{c}(t), \dot{y}_{c}(t) \in \mathcal{L}_{\infty}$. Based on the boundedness of the aforementioned signals, we can take the time derivative of (18) and show that $\ddot{z}_{d}(t) \in \mathcal{L}_{\infty}$ (see the Appendix for explicit details). Standard signal chasing arguments can now be used to show that all remaining signals are bounded.

From (14) and (34), it is easy to show that $z_{d}(t), \tilde{z}(t) \in \mathcal{L}_{2}$; hence, since $z_{d}(t)$ and $\tilde{z}(t)$ are uniformly continuous, we can use (14) and a corollary to Barbalat's Lemma [25] to show that $\lim _{t \rightarrow \infty} z_{d}(t), \tilde{z}(t), z(t)=0$. Next, since $\ddot{z}_{d}(t) \in \mathcal{L}_{\infty}$, we know that $\dot{z}_{d}(t)$ is uniformly continuous. Since we know that $\lim _{t \rightarrow \infty} z_{d}(t)=0$ and $\dot{z}_{d}(t)$ is uniformly continuous, we can use the following equality:

$$
\lim _{t \rightarrow \infty} \int_{0}^{t} \frac{d}{d \tau}\left(z_{d}(\tau)\right) d \tau=\lim _{t \rightarrow \infty} z_{d}(t)+\text { Constant }
$$

and Barbalat's Lemma [25] to conclude that $\lim _{t \rightarrow \infty} \dot{z}_{d}(t)=0$. Based on the fact that $\lim _{t \rightarrow \infty} z_{d}(t), \dot{z}_{d}(t)=0$, it is straightforward from (17) and (18) to see that $\lim _{t \rightarrow \infty} w A^{T}=0$. Finally, based on (10) and (29) we can conclude that $\lim _{t \rightarrow \infty} w(t)=0$. The global asymptotic result given in (28) can now be directly obtained from (35).

\section{Global Exponential Tracking Analysis}

In the previous section, we utilized a straightforward Lyapunov analysis and Barbalat's Lemma to prove global asymptotic position/orientation tracking. Since we have established that all signals in the closed-loop system are bounded, we now illustrate how the nonlinear closed-loop error system formulated in the previous section can be represented as a linear timevarying system as similarly done for closed-loop adaptive control systems (see [19]). This linear time-varying representation allows us to develop a PE condition on the desired reference trajectory that promulgates a global exponential tracking result.

\section{A. Error System Development}

To formulate the nonlinear closed-loop error development given in the previous section as a linear time-varying system, we first define the states of the system, denoted by $x(t) \in \Re^{5}$, as follows:

$$
x=\left[\begin{array}{ll}
p^{T} & w
\end{array}\right]^{T}
$$


where the auxiliary signal $p(t) \in \Re^{4}$ is defined as

$$
p=\left[\begin{array}{ll}
z_{d}^{T} & \tilde{z}^{T}
\end{array}\right]^{T}
$$

and $w(t), z_{d}(t)$, and $z(t)$ were defined in (6). In addition, we rewrite the closed-loop dynamics for $w(t)$, into a more convenient form by substituting (16) for only the second occurrence of $u_{a}(t)$ in (21) and then utilizing (14), the skew symmetry of $J$ defined in (9), and the fact that $J^{T} J=I_{2}$ to yield

$$
\dot{w}=u_{a}^{T} J \tilde{z}-k_{1} w z_{d}^{T} z_{d}+A\left(z_{d}-\tilde{z}\right)-u_{c}^{T} J\left(z_{d}-\tilde{z}\right) .
$$

Based on (39) and the definition of $p(t)$ given in (38), we can now obtain a convenient expression for the dynamics of $w(t)$ as follows:

$$
\dot{w}=B_{1}^{T} p
$$

where $B_{1}(t) \in \Re^{4}$ is defined as

$$
B_{1}=\left[\begin{array}{c}
-k_{1} w z_{d}^{T}+A-u_{c}^{T} J \\
u_{a}^{T} J-A+u_{c}^{T} J
\end{array}\right]
$$

In order to express the closed-loop error system for $\tilde{z}(t)$ in a form that facilitates the linear system representation, we substitute (17) into (26) to obtain the following expression:

$$
\dot{\tilde{z}}=\left(w J \Omega_{1}-k_{1} w^{2} I_{2}\right) z_{d}+\left(\left(I_{2}+2 w J\right)^{-1} A^{T}\right) w-k_{3} \tilde{z}
$$

It is now a straightforward matter to take the time derivative of (38) and make appropriate substitutions from (23) and (42) to express the closed-loop error system for $p(t)$ as follows:

$$
\dot{p}=A_{0} p+B_{2} w
$$

where the auxiliary terms $A_{0}(t) \in \Re^{4 \times 4}$ and $B_{2}(t) \in \Re^{4}$ are defined as

$$
A_{0}=\left[\begin{array}{cc}
\left(k_{1}\left(w^{2}-z_{d}^{T} z_{d}\right)-k_{2}\right) I_{2}+J \Omega_{2} & 0_{2 \times 2} \\
w J \Omega_{1}-k_{1} w^{2} I_{2} & -k_{3} I_{2}
\end{array}\right]
$$

and

$$
B_{2}=\left[\begin{array}{c}
-\left(\left(I_{2}+2 w J\right)^{-1} A^{T}\right) \\
\left(\left(I_{2}+2 w J\right)^{-1} A^{T}\right)
\end{array}\right]
$$

respectively, $0_{n \times m}$ represents the $n \times m$ zero matrix, and $I_{2}$ represents the $2 \times 2$ identity matrix. The final linear time-varying representation is obtained by taking the time derivative of (37) and then utilizing (40) and (43) to obtain

$$
\begin{aligned}
& \dot{x}=A_{1} x \\
& y=C x
\end{aligned}
$$

where the matrix $A_{1}(t) \in \Re^{5 \times 5}$ is defined as

$$
A_{1}=\left[\begin{array}{cc}
A_{0} & B_{2} \\
B_{1}^{T} & 0
\end{array}\right]
$$

the matrix $C \in \Re^{5 \times 5}$ is defined as

$$
C=\left[\begin{array}{cc}
D & 0_{4 \times 1} \\
0_{1 \times 4} & 0
\end{array}\right]
$$

and the submatrix $D \in \Re^{4 \times 4}$ is defined as

$$
D=\left[\begin{array}{cc}
\sqrt{k_{2}} I_{2} & 0_{2 \times 2} \\
0_{2 \times 2} & \sqrt{k_{3}} I_{2}
\end{array}\right] .
$$

Remark 1: In the subsequent exponential stability proof, we will utilize the fact that (34) can be rewritten as

$$
\dot{V} \leq-x^{T} C^{T} C x
$$

hence, (50) provides the motivation for the structure of the matrix $C$ defined in (48). The subsequent stability analysis also utilizes the fact that all the signals in the time-varying system given by (46) are bounded as illustrated by Theorem 1; furthermore, the stability analysis requires that $B_{2}(t)$ defined in (45) be differentiable. Based on the proof of Theorem 1, it is straightforward to show that $\dot{B}_{2}(t)$ exists and is a bounded matrix.

\section{B. Stability Analysis}

Before we state the exponential stability result, we present two lemmas that are used during the proof of the main result.

Lemma 1: If the reference angular velocity $v_{r 2}(t)$ defined in (5) is selected according to the following expression:

$$
\int_{t}^{t+\delta_{1}} v_{r 2}^{2}(\sigma) d \sigma \geq \xi_{1}
$$

[i.e., if the reference angular velocity is selected to be persistently exciting $(\mathrm{PE})]$ then the observability grammian for the system given in (46), defined as

$$
W(t, t+\delta)=\int_{t}^{t+\delta} \Phi^{T}(\tau, t) C^{T} C \Phi(\tau, t) d \tau
$$

satisfies the following inequality:

$$
W(t, t+\delta) \geq \gamma I_{5}
$$

for all $t \geq 0$, where $\delta, \delta_{1}, \xi_{1}, \gamma \in \Re^{1}$ are positive constants, $\Phi(\tau, t) \in \Re^{5 \times 5}$ denotes the state transition matrix for (46), $I_{n}$ represents the $n \times n$ identity matrix, and $C$ was defined in (48).

Proof: To prove Lemma 1, we note that a closed-form expression for the state transition matrix of (46) is difficult to find; thus, we employ the fact that the pair $\left(A_{1}(t), C\right)$ of (46) is uniformly observable (UO) if and only if the pair $\left(A_{1}(t)-\right.$ $K(t) C, C$ ) is $\mathrm{UO}$ (see [15] for an explicit proof) where $K(t) \in$ $\Re^{5 \times 5}$ is treated as a design matrix. To facilitate the analysis, we construct $K(t)$ to be a continuous, bounded matrix as follows:

$$
K=\left[\begin{array}{cc}
A_{0} D^{-1} & 0_{4 \times 1} \\
B_{1}^{T} D^{-1} & 0
\end{array}\right] .
$$

Based on the definition of $K(t)$ given in (54), we have

$$
A_{1}-K C=\left[\begin{array}{cc}
0_{4 \times 4} & B_{2} \\
0_{1 \times 4} & 0
\end{array}\right] ;
$$

hence, the state transition matrix for the pair $\left(A_{1}(t)\right.$ $K(t) C, C)$, denoted by $\Phi_{1}(\tau, t) \in \Re^{5 \times 5}$, can be calculated as follows:

$$
\Phi_{1}=\left[\begin{array}{cc}
I_{4} & \int_{t}^{\tau} B_{2}(\sigma) d \sigma \\
0_{1 \times 4} & 1
\end{array}\right] .
$$


Given the following definition for the observability grammian for the pair $\left(A_{1}(t)-K(t) C, C\right)$

$$
W_{1}\left(t, t+\delta_{2}\right)=\int_{t}^{t+\delta_{2}} \Phi_{1}^{T}(\tau, t) C^{T} C \Phi_{1}(\tau, t) d \tau
$$

we can substitute (48) and (56) into (57) to calculate (58) (shown at the bottom of the page), where $\delta_{2} \in \Re^{1}$ is a positive constant.

To facilitate further analysis, we note that $\int_{t}^{t+\delta_{1}} B_{2}^{T}(\sigma) B_{2}(\sigma) d \sigma$ can be rewritten as follows:

$$
\begin{aligned}
\int_{t}^{t+\delta_{1}} & B_{2}^{T}(\sigma) B_{2}(\sigma) d \sigma \\
=2 & {\left[\int _ { t } ^ { t + \delta _ { 1 } } \frac { 1 } { ( 1 + 4 w ^ { 2 } ) ^ { 2 } } \left(\left(-2 v_{r 1} \frac{\sin \left(z_{1}\right)}{z_{1}}+4 w v_{r 2}\right)^{2}\right.\right.} \\
& \left.\left.\quad+\left(4 w v_{r 1} \frac{\sin \left(z_{1}\right)}{z_{1}}+2 v_{r 2}\right)^{2}\right) d \sigma\right]
\end{aligned}
$$

After some algebraic manipulation, we note that (59) can be simplified as follows:

$$
\begin{aligned}
& \int_{t}^{t+\delta_{1}} B_{2}^{T}(\sigma) B_{2}(\sigma) d \sigma \\
& \quad=8 \int_{t}^{t+\delta_{1}} \frac{1}{\left(1+4 w^{2}\right)}\left(v_{r 1}^{2}\left(\frac{\sin \left(z_{1}\right)}{z_{1}}\right)^{2}+v_{r 2}^{2}\right) d \sigma .
\end{aligned}
$$

Next, since $w(t), z(t), v_{r}(t) \in \mathcal{L}_{\infty}$, we can select positive constants $\zeta_{1}, \gamma_{1} \in \Re^{1}$ such that $\int_{t}^{t+\delta_{1}} B_{2}^{T}(\sigma) B_{2}(\sigma) d \sigma$ can be lower bounded as follows:

$$
\int_{t}^{t+\delta_{1}} B_{2}^{T}(\sigma) B_{2}(\sigma) d \sigma \geq \zeta_{1} \int_{t}^{t+\delta_{1}} v_{r 2}^{2} d \sigma \geq \gamma_{1}
$$

where the assumption given in (51) was utilized. Given the definition for $W_{1}\left(t, t+\delta_{2}\right)$ in (58), the fact that $B_{2}(t)$ and $\dot{B}_{2}(t)$ are bounded, and the fact that $\int_{t}^{t+\delta_{1}} B_{2}^{T}(\sigma) B_{2}(\sigma) d \sigma$ satisfies (61), we can apply [19, Lemma 13.4] to (58) to show that there exists some positive constant $\gamma_{2} \in \Re^{1}$ such that

$$
W_{1}\left(t, t+\delta_{2}\right) \geq \gamma_{2} I_{5}
$$

hence, the pair $\left(A_{1}(t)-K(t) C, C\right)$ is UO. Since the pair $\left(A_{1}(t)-K(t) C, C\right)$ is $\mathrm{UO}$, then the pair $\left(A_{1}(t), C\right)$ is $\mathrm{UO}$ (see [15, Lemma 4.8.1] for an explicit proof); hence, by the definition of uniform observability (see [2]), the result given in (53) can now be directly obtained.

Lemma 2: Let $V(x, t) \in \Re^{1}$ be a continuously differentiable function such that

$$
\gamma_{a}\|x\|^{2} \leq V(x, t) \leq \gamma_{b}\|x\|^{2}
$$

$$
\dot{V}(x, t) \leq 0 \&(64)
$$

and

$$
\int_{t}^{t+\delta} \dot{V}(\phi(x, t, \tau), \tau) d \tau \leq-\gamma_{c} V(x, t) \quad \text { for } t \geq 0
$$

where $\gamma_{a}, \gamma_{b}, \gamma_{c}, \delta \in \Re^{1}$ are positive constants and $\phi(x, t, \tau)$ are the solution of the system that starts at $(x, t)$. If (63)-(65) hold globally, then the system is globally exponentially stable in the sense that

$$
\|x(t)\| \leq \alpha_{0} \exp \left(-\beta_{0} t\right)
$$

for some positive constants $\alpha_{0}, \beta_{0} \in \Re^{1}$.

Proof: See [19, Theorem 4.5].

Remark 2: Note that since (66) is an exponential envelope originating at $\alpha_{0}$ which need not be proportional to $\|x(0)\|$, the result does not adhere to the standard definition of global exponential stability (see the discussion in [19] and [23]); however, for any initial condition, $x(t)$ exponentially converges to zero.

Theorem 2: The position and orientation tracking errors defined in (7) are globally exponentially stable in the sense that

$$
|\tilde{x}(t)|,|\tilde{y}(t)|,|\tilde{\theta}(t)| \leq \alpha_{1} \exp \left(-\beta_{1} t\right)
$$

for some positive scalar constants $\alpha_{1}$ and $\beta_{1}$ provided that the reference angular velocity satisfies (51).

Proof: To prove Theorem 2, we define the nonnegative function $V_{2}(x) \in \Re^{1}$ as follows:

$$
V_{2}(x, t)=\frac{1}{2} x^{T} x
$$

where $x(t)$ was defined in (37). Based on (30), (34), (37), (38), (48), (49), (68), and the proof for Theorem 1, the time derivative of (68) can be expressed as follows:

$$
\dot{V}_{2}(x, t) \leq-x^{T} C^{T} C x
$$

where $C$ was defined in (48). After integrating (69), we obtain the following expression:

$$
\begin{aligned}
& \int_{t}^{t+\delta} \dot{V}_{2}(\phi(x, \tau, t), \tau) d \tau \\
& \quad \leq-x^{T}\left[\int_{t}^{t+\delta} \Phi^{T}(\tau, t) C^{T} C \Phi(\tau, t) d \tau\right] x
\end{aligned}
$$

where we have use the fact that $\phi(x, \tau, t) \in \Re^{5}$, which denotes the solution to the linear system defined in (46) that starts at $(x, t)$, can be expressed as follows: [3]

$$
\phi(x, \tau, t)=\Phi(\tau, t) x(t)
$$

$$
W_{1}\left(t, t+\delta_{2}\right)=\int_{t}^{t+\delta_{2}}\left[\begin{array}{cc}
D^{T} D & D^{T} D \int_{t}^{\tau} B_{2}(\sigma) d \sigma \\
\int_{t}^{\tau} B_{2}^{T}(\sigma) d \sigma D^{T} D & \int_{t}^{\tau} B_{2}^{T}(\sigma) d \sigma D^{T} D \int_{t}^{\tau} B_{2}(\sigma) d \sigma
\end{array}\right] d \tau
$$


and $\Phi(\tau, t) \in \Re^{5 \times 5}$ denotes the state transition matrix for (46). After noting that the bracketed term in (70a) is equal to $W(t, t+$ $\delta$ ), defined in (52), we can utilize (53), (68), Lemma 1, and (51) to show that

$$
\int_{t}^{t+\delta} \dot{V}_{2}(\phi(x, \tau, t), \tau) d \tau \leq-2 \gamma V_{2}(x, t) .
$$

From (68), (69), and (72), it is clear that the conditions given in Lemma 2 are globally satisfied; hence,

$$
\|x(t)\| \leq \alpha_{2} \exp \left(-\beta_{2} t\right)
$$

where $\alpha_{2}$ and $\beta_{2} \in \Re^{1}$ are positive constants. The global exponential result given in (67) can now be directly obtained from (14), (35), (37), and (38).

Remark 3: We note that with the achievement of exponential position/orientation tracking as seen in (67), a certain degree of robustness is acquired for the proposed tracking controller. That is, exponentially stable systems inherently have the ability to tolerate a greater degree of uncertainty in the form of unknown parameters, external disturbances, unmodeled dynamics, etc. as compared to an asymptotically stable system. For a more detailed discussion on the theorems and analysis concerning the robustness of exponentially stable systems, see [27] and the references therein.

Remark 4: Some examples of persistently exciting reference trajectories include: 1) $v_{r 2} \neq 0$; 2) $\lim _{t \rightarrow \infty} v_{r 2}=c_{1} \neq 0$ (e.g., a circle trajectory can be exponentially tracked); and 3) $v_{r 2}=\sin (t)$. In addition, we note that since $\lim _{t \rightarrow \infty} z(t)=0$ (see Theorem 1), then there exists some time, denoted by $t_{p}$, such that

$$
\left|z_{1}(t)\right|<\frac{\pi}{2} \quad \forall t>t_{p}
$$

thus,

$$
\frac{\sin \left(z_{1}\right)}{z_{1}}>\frac{2}{\pi} \quad \forall t>t_{p}
$$

Based on (60) and (75), we can rewrite (61) as follows:

$$
\begin{aligned}
& \int_{t}^{t+\delta_{1}} B_{2}^{T}(\sigma) B_{2}(\sigma) d \sigma \\
& \quad \geq \zeta_{1}\left(\frac{2}{\pi}\right)^{2} \int_{t}^{t+\delta_{1}}\left(v_{r 1}^{2}+v_{r 2}^{2}\right) d \sigma \quad \forall t>t_{p}
\end{aligned}
$$

hence, if the reference condition given in (51) is modified as given below

$$
\int_{t}^{t+\delta_{1}}\left\|v_{r}(\sigma)\right\|^{2} d \sigma \geq \xi_{1} \quad \forall t>t_{p}
$$

(i.e., if either the linear or angular reference velocity is selected to be $\mathrm{PE}$ ), then

$$
|\tilde{x}(t)|,|\tilde{y}(t)|,|\tilde{\theta}(t)| \leq \alpha_{1} \exp \left(-\beta_{1} t\right), \quad \forall t>t_{p}
$$

The inequality given in (78) indicates that if (77) is satisfied, then there is some time during the transient after which the asymptotic tracking result becomes an exponential tracking result; hence, many different types of geometric trajectories can be exponentially tracked after some finite time (e.g., lines).

\section{Setpoint Extension}

Many of the previously proposed tracking controllers do not reduce to the regulation problem because of technical restrictions placed on the reference trajectory similar to that given in (29). In this section, we illustrate how the kinematic tracking controller proposed in the previous section can be slightly modified to ensure global asymptotic position and orientation regulation [i.e., $k_{2}$ given in (18) and (19) is set equal to zero]. Since this new control objective is now targeted at the regulation problem, the desired position and orientation vector, denoted by $q_{r}=\left[\begin{array}{lll}x_{r c} & y_{r c} & \theta_{r}\end{array}\right]^{T} \in \Re^{3}$ and originally defined in (5), is now assumed to be an arbitrary desired constant vector. Based on the fact that $q_{r}$ is now defined as a constant vector, it is straightforward to see that $v_{r}(t)$, given in (5), and consequently $A\left(z, v_{r}, t\right)$ and $u_{c}(t)$ defined in (10) and (17), respectively, are now set to zero for the regulation control problem. As a result of the new control objective, we also note that the auxiliary variable $u(t)$ originally defined in (11), is now defined as follows:

$$
u=T^{-1} v \quad v=T u
$$

where the matrix $T$ was defined in (12).

\section{A. Stability Analysis}

Theorem 3: The kinematic controller given by (15), (16), (18)-(20) with $k_{2}=0$, ensures global asymptotic regulation in the sense that

$$
\lim _{t \rightarrow \infty} \tilde{x}(t), \tilde{y}(t), \tilde{\theta}(t)=0
$$

where the position and orientation setpoint errors were defined in (7).

Proof: To prove Theorem 3, we take the time derivative of the nonnegative function given in (30), and then substitute (22), (27), and (18) in the resulting expression (where $A\left(z, v_{r}, t\right)$, $u_{c}(t)$, and $k_{2}$ are all equal to zero for the regulation problem) and follow the proof of Theorem 1 to obtain the following expression

$$
\begin{aligned}
\dot{V}= & w\left[u_{a}^{T} J \tilde{z}-k_{1} w z_{d}^{T} z_{d}\right]+z_{d}^{T} J \Omega_{2} z_{d} \\
& +k_{1} z_{d}^{T}\left(w^{2}-z_{d}^{T} z_{d}\right) z_{d}+\tilde{z}^{T}\left[w J u_{a}-k_{3} \tilde{z}\right] .
\end{aligned}
$$

After utilizing the skew symmetry property of $J$ defined in (9), making use of the fact that $J^{T}=-J$, and then cancelling common terms, we can express $\dot{V}(t)$ of (81) as follows:

$$
\dot{V}=-k_{1}\left\|z_{d}\right\|^{4}-k_{3}\|\tilde{z}\|^{2} \text {. }
$$

Based on the same arguments as given for the proof of Theorem 1, we can show that all signals remain bounded during closed-loop operation, and that $\lim _{t \rightarrow \infty} z_{d}(t), \tilde{z}(t), z(t)=0$. Since (30) is a positive, radially unbounded function with a negative semi-definite time derivative as shown in (82), we can also conclude that $\lim _{t \rightarrow \infty} V\left(w, z_{d}, \tilde{z}, t\right)=c_{1}$ where $c_{1} \in \Re^{1}$ is a constant. Furthermore, since $\lim _{t \rightarrow \infty} z_{d}(t), \tilde{z}(t)=0$, it is 
straightforward from (30) that $\lim _{t \rightarrow \infty} w^{2}=c_{2}$ where $c_{2} \in \Re^{1}$ is a nonnegative constant.

In order to facilitate further analysis, we define the following nonnegative function $v_{0}\left(z_{d}, t\right) \in \Re^{1}$ as follows:

$$
v_{0}=\frac{1}{2} z_{d}^{T} z_{d} \quad v_{0}(0)=\frac{\beta}{2}
$$

where $\beta$ was defined in (18). Based on (83) and the fact that $\lim _{t \rightarrow \infty} z_{d}(t)=0$, it is straightforward to see that $\lim _{t \rightarrow \infty} v_{0}(t)=0$; hence, for all $\varepsilon_{1}>0$, we have that

$$
v_{0}(t)<\varepsilon_{1} \quad \forall t>T_{o 1}\left(\varepsilon_{1}\right)
$$

where $\varepsilon_{1}, T_{o 1}\left(\varepsilon_{1}\right) \in \Re^{1}$ are positive constants. After taking the time derivative of $v_{0}(t)$ given in (83), we have

$$
\dot{v}_{0}=-4 k_{1} v_{0}^{2}+2 k_{1} w^{2} v_{0}
$$

where (18) and the skew symmetry property of $J$ defined in (9) have been used. After dividing (85) by $v_{0}^{2}$ and then integrating the resulting equation, we have

$$
\frac{1}{v_{0}(t)}-\frac{1}{v_{0}(0)}=\int_{0}^{t} 4 k_{1} d \sigma-\int_{0}^{t} \frac{2 k_{1} w^{2}(\sigma)}{v_{0}(\sigma)} d \sigma .
$$

Based on the fact that

$$
\int_{0}^{t} \frac{2 k_{1} w^{2}(\sigma)}{v_{0}(\sigma)} d \sigma \geq 0
$$

we can rearrange (86) to obtain a lower bound for $v_{0}(t)$ as follows

$$
v_{0}(t) \geq \frac{1}{\frac{1}{v_{0}(0)}+4 k_{1} t} .
$$

Now, we use (84), (88), the structure of (85), and the fact that $\lim _{t \rightarrow \infty} v_{0}(t)=0$ to prove by contradiction that $\lim _{t \rightarrow \infty} w^{2}(t)=0$. To facilitate the proof by contradiction, we assume that $\lim _{t \rightarrow \infty} w^{2}(t)=c_{2}>0$; hence, for all $\varepsilon_{2}>0$, we have that

$$
\left|w^{2}-c_{2}\right|<\varepsilon_{2} \quad \forall t>T_{o 2}\left(\varepsilon_{2}\right)
$$

where $\varepsilon_{2}, T_{o 2}\left(\varepsilon_{2}\right) \in \Re^{1}$ are positive constants. If we select $\varepsilon_{1}=\left(c_{2} / 8\right)$ and $\varepsilon_{2}=\left(c_{2} / 2\right)$, then from (84), (88), and (89), we have that

$$
0 \leq v_{0}<\frac{c_{2}}{8} \quad \forall t>T_{01}\left(\frac{c_{2}}{8}\right)
$$

and

$$
\frac{c_{2}}{2}<w^{2}<\frac{3 c_{2}}{2} \quad \forall t>T_{02}\left(\frac{c_{2}}{2}\right) .
$$

Furthermore, if we select $T_{0} \in \Re^{1}$ as

$$
T_{0}=\max \left(T_{01}\left(\frac{c_{2}}{8}\right), T_{02}\left(\frac{c_{2}}{2}\right)\right)
$$

then from (85), (90), and (91), we can conclude that $\dot{v}_{0}(t)$ is nonnegative as shown below

$$
\begin{aligned}
\dot{v}_{0}(t) & \geq-4 k_{1} v_{0}\left(\frac{c_{2}}{8}\right)+2 k_{1}\left(\frac{c_{2}}{2}\right) v_{0} \\
& \geq \frac{1}{2} k_{1} c_{2} v_{0} \geq 0 \quad \forall t>T_{0} .
\end{aligned}
$$

Now, note that from (88), we have that

$$
v_{0}\left(T_{0}\right) \geq \frac{1}{\frac{1}{v_{0}(0)}+4 k_{1} T_{0}} .
$$

In addition, we note that

$$
v_{0}(t)=v_{0}\left(T_{0}\right)+\int_{T_{0}}^{t} \dot{v}_{0}(\sigma) d \sigma
$$

which can be lower bounded as follows:

$$
v_{0}(t) \geq v_{0}\left(T_{0}\right)
$$

as a result of (93). Based on (94) and (96), we can conclude that

$$
v_{0}(t) \geq \frac{1}{\frac{1}{v_{0}(0)}+4 k_{1} T_{0}}
$$

however, (97) is a contradiction to the fact that $\lim _{t \rightarrow \infty} v_{0}(t)=$ 0 . Since the assumption that $\lim _{t \rightarrow \infty} w^{2}(t)=c_{2}>0$ leads to a contradiction, we can conclude that $\lim _{t \rightarrow \infty} w^{2}(t)=0$; hence, $\lim _{t \rightarrow \infty} w(t)=0$. Finally, since $\lim _{t \rightarrow \infty} z_{d}(t), \tilde{z}(t)$, $w(t)=0$, the global asymptotic result given in (80) can now be directly obtained from (35).

\section{DYNAMIC EXTENSION}

Practical issues (e.g., robustness to uncertainty in the dynamic model) provide motivation to include the dynamic model as part of the overall control problem. As a result of this motivation, we describe the dynamic model of the WMR and then demonstrate how the integrator backstepping technique can be utilized to develop a tracking controller that is based on the composite kinematic/dynamic model. Specifically, in the following section, we present an adaptive controller that achieves global asymptotic tracking control despite parametric uncertainty in the dynamic model.

\section{A. WMR Dynamic Model}

The dynamic model for the kinematic wheel can be expressed in the following form:

$$
M \dot{v}+F(v)=B \tau
$$

where

$\dot{v}(t) \in \Re^{2} \quad$ time derivative of $v(t)$ defined in (4);

$M \in \Re^{2 \times 2} \quad$ constant inertia matrix;

$F(v) \in \Re^{2} \quad$ friction effects;

$\tau(t) \in \Re^{2} \quad$ torque input vector;

$B \in \Re^{2 \times 2} \quad$ input matrix that governs torque transmission.

To facilitate the subsequent control design, we premultiply (98) by $T^{T}$ defined in (12), and substitute (11) and (12) for $v(t)$ to obtain the following convenient dynamic model

$$
\bar{M} \dot{u}+\bar{V}_{m} u+\bar{N}=\bar{B} \tau
$$

where

$$
\begin{aligned}
\bar{M} & =T^{T} M T, \bar{V}_{m}=T^{T} M \dot{T} \\
\bar{B} & =T^{T} B, \bar{N}=T^{T}(F(T u+\Pi)+M \dot{\Pi}) .
\end{aligned}
$$


The dynamic equation of (99) exhibits the following properties [20] which will be employed during the subsequent control development and stability analysis.

Property 1: The transformed inertia matrix $\bar{M}$ is symmetric, positive definite, and satisfies the following inequalities:

$$
m_{1}\|\xi\|^{2} \leq \xi^{T} \bar{M} \xi \leq m_{2}(z, w)\|\xi\|^{2} \quad \forall \xi \in \Re^{2}
$$

where

$$
\begin{array}{ll}
m_{1} & \text { known positive constant; } \\
m_{2}(z, w) \in & \begin{array}{l}
\text { known, positive bounding function which is } \\
\text { assumed to be bounded provided its argu- } \\
\Re^{1}
\end{array} \\
\text { ments are bounded; } \\
\text { standard Euclidean norm. }
\end{array}
$$

Property 2: A skew-symmetric relationship exists between the transformed inertia matrix and the auxiliary matrix $\bar{V}_{m}$ as follows:

$$
\xi^{T}\left(\frac{1}{2} \dot{\bar{M}}-\bar{V}_{m}\right) \xi=0 \quad \forall \xi \in \Re^{2}
$$

where $\dot{\bar{M}}$ represents the time derivative of the transformed inertia matrix.

Property 3: The robot dynamics given in (99) can be linearly parameterized as follows:

$$
Y_{o} \vartheta=\bar{M} \dot{u}+\bar{V}_{m} u+\bar{N}
$$

where $\vartheta \in \Re^{p}$ contains the unknown constant mechanical parameters (i.e., inertia, mass, and friction effects) and $Y_{o}(\dot{u}, u, t) \in \Re^{n \times p}$ is the known regression matrix.

\section{B. Control Formulation}

Based on the desire to incorporate the dynamic model in the control design, our new control objective is to design an adaptive tracking controller for the transformed WMR model given by (99). To this end, we reformulate the kinematic controller given in (15) as a desired signal as follows:

$$
u_{d}=u_{a}-k_{3} z+u_{c}
$$

where $u_{d}(t) \in \Re^{2}$ denotes the desired kinematic control signal. Furthermore, based on the transformed dynamic model given by (99) and the subsequent stability analysis, we design the control torque input $\tau(t)$ as follows:

$$
\tau=\bar{B}^{-1}\left(Y \hat{\vartheta}+K_{a} \eta+J z w+\tilde{z}\right)
$$

where $K_{a} \in \Re^{2 \times 2}$ is a positive definite, diagonal control gain matrix and $\eta(t) \in \Re^{2}$ is a tracking error signal defined as follows:

$$
\eta=u_{d}-u
$$

$\hat{\vartheta}(t) \in \Re^{p}$ denotes the parameter estimate of $\vartheta$, and is calculated on-line via the following dynamic update law:

$$
\dot{\hat{\vartheta}}=\Gamma Y^{T} \eta
$$

and the regression matrix $Y\left(\dot{u}_{d}, u_{d}, u, t\right) \in \Re^{n \times p}$ is defined as follows:

$$
Y \vartheta=\bar{M} \dot{u}_{d}+\bar{V}_{m} u_{d}+\bar{N}
$$

To quantify the performance of the adaptation algorithm, we define the parameter estimation error signal, denoted by $\tilde{\vartheta}(t) \in$ $\Re^{p}$, as follows:

$$
\tilde{\vartheta}=\vartheta-\hat{\vartheta}
$$

\section{Error System Development}

To facilitate the closed-loop error system development for $w(t)$, we inject the desired kinematic control input $u_{d}(t)$ into the open-loop dynamics of $w(t)$ given by (8) by adding and subtracting the term $u_{d}^{T} J z$ to the right-side of (8) and utilizing (106) to obtain the following expression:

$$
\dot{w}=\eta^{T} J_{z}-u_{d}^{T} J_{z}+A z .
$$

After substituting (104) for $u_{d}(t)$, adding and subtracting $u_{a}^{T} J z_{d}$ to the resulting expression, utilizing (14), and exploiting the skew symmetry of $J$ defined in (9), we can rewrite the dynamics for $w(t)$ as follows:

$$
\dot{w}=\eta^{T} J z+u_{a}^{T} J \tilde{z}-u_{a}^{T} J_{d}+A z-u_{c}^{T} J z .
$$

By utilizing the same operations illustrated in the kinematic control development, we can obtain the final expression for the closed-loop error system for $w(t)$ as follows:

$$
\begin{aligned}
\dot{w}= & \eta^{T} J z+u_{a}^{T} J \tilde{z}-k_{1} w z_{d}^{T} z_{d} \\
& +A z+2 w A\left(I_{2}+2 w J^{T}\right)^{-1} J z .
\end{aligned}
$$

To determine the closed-loop error system for $\tilde{z}(t)$, we take the time derivative of (14), substitute (18) for $\dot{z}_{d}(t)$, and then substitute (8) for $\dot{z}(t)$ to obtain

$$
\begin{aligned}
\dot{\tilde{z}}= & \left(k_{1}\left(w^{2}-z_{d}^{T} z_{d}\right)-k_{2}\right) z_{d} \\
& +J \Omega_{2} z_{d}+\frac{1}{2} u_{c}+\eta-u_{d}
\end{aligned}
$$

where (106) was utilized and the desired kinematic control signal $u_{d}(t)$ was injected by adding and subtracting $u_{d}(t)$ to the right-side of (113). Based on the expression given in (113), we can obtain the final expression for the closed-loop error system for $\dot{\tilde{z}}(t)$ as shown below

$$
\dot{\tilde{z}}=w J u_{a}-k_{3} \tilde{z}+\left(I_{2}+2 w J\right)^{-1} w A^{T}+\eta
$$

by following the same procedure as described in the kinematic control development.

In order to develop the closed-loop error system for $\eta(t)$, we take the time derivative of (106), premultiply the resulting expression by the transformed inertia matrix, and substitute for $\dot{u}(t)$ from (99) to obtain the following expression:

$$
\bar{M} \dot{\eta}=-\bar{V}_{m} \eta+Y \vartheta-\bar{B} \tau
$$

where $\bar{V}_{m} u_{d}$ was added and subtracted to the right-side of (115) and (108) was utilized. After substituting (105) into (115), we obtain the following expression for the closed-loop error system:

$$
\bar{M} \dot{\eta}=-\bar{V}_{m} \eta+Y \tilde{\vartheta}-K_{a} \eta-J z w-\tilde{z}
$$

where (109) was utilized. 


\section{Stability Analysis}

Theorem 4: The controller given by (16)-(20), (104), (105), and (107) ensures global asymptotic tracking in the sense that

$$
\lim _{t \rightarrow \infty} \tilde{x}(t), \tilde{y}(t), \tilde{\theta}(t)=0
$$

provided the reference trajectory is selected so that

$$
\lim _{t \rightarrow \infty}\left\|v_{r}\right\| \neq 0
$$

Proof: To prove Theorem 4, we define the following nonnegative function denoted by $V_{3}\left(w, z_{d}, \tilde{z}, \eta, \tilde{\vartheta}, t\right) \in \Re^{1}$ as follows:

$$
\begin{aligned}
V_{3}(t)= & \frac{1}{2} w^{2}+\frac{1}{2} z_{d}^{T} z_{d}+\frac{1}{2} \tilde{z}^{T} \tilde{z} \\
& +\frac{1}{2} \eta^{T} \bar{M} \eta+\frac{1}{2} \tilde{\vartheta}^{T} \Gamma^{-1} \tilde{\vartheta}
\end{aligned}
$$

After taking the time derivative of (119) and making the appropriate substitutions from (23), (107), (112), (114), and (116), and utilizing the fact that $\dot{\tilde{\vartheta}}=-\dot{\hat{\vartheta}}$, we obtain the following expression:

$$
\begin{aligned}
\dot{V}_{3}= & w\left[\eta^{T} J z+u_{a}^{T} J \tilde{z}-k_{1} w z_{d}^{T} z_{d}\right] \\
& +w\left[A z+2 w A\left(I_{2}+2 w J^{T}\right)^{-1} J z\right] \\
& +z_{d}^{T}\left[\left(k_{1}\left(w^{2}-z_{d}^{T} z_{d}\right)-k_{2}\right) z_{d}\right] \\
& +z_{d}^{T}\left[J \Omega_{2} z_{d}-\left(I_{2}+2 w J\right)^{-1} w A^{T}\right] \\
& +\tilde{z}^{T}\left[-k_{3} \tilde{z}+\left(I_{2}+2 w J\right)^{-1} w A^{T}\right] \\
& +\tilde{z}^{T}\left[w J u_{a}+\eta\right]+\frac{1}{2} \eta^{T} \dot{\bar{M}} \eta-\tilde{\vartheta}^{T}\left[Y^{T} \eta\right] \\
& +\eta^{T}\left[Y \tilde{\vartheta}-J z w-\tilde{z}-K_{a} \eta-\bar{V}_{m} \eta\right] .
\end{aligned}
$$

After utilizing (102) and canceling common terms, we can use the same procedure as presented in the proof of Theorem 1 to obtain the final expression for $\dot{V}_{3}(t)$ as follows:

$$
\dot{V}_{3}=-k_{1}\left\|z_{d}\right\|^{4}-k_{2} z_{d}^{T} z_{d}-k_{3} \tilde{z}^{T} \tilde{z}-\eta^{T} K_{a} \eta .
$$

Based on (119) and (121), we can conclude that $V_{3}(t) \in \mathcal{L}_{\infty}$; thus, $w(t), z_{d}(t), \tilde{z}(t), \eta(t), \tilde{\vartheta}(t) \in \mathcal{L}_{\infty}$. Since $w(t), z_{d}(t)$, $\tilde{z}(t), \eta(t), \tilde{\vartheta}(t) \in \mathcal{L}_{\infty}$, we can utilize (107), and the same arguments given in the proof for Theorem 1 to conclude that $A(t)$, $z(t), u_{d}(t), u_{a}(t), u_{c}(t), \Omega_{1}(t), \Omega_{2}(t), \dot{z}_{d}(t), \dot{w}(t), \dot{\tilde{z}}(t), \tilde{\vartheta}(t)$, $\hat{\vartheta}(t) \in \mathcal{L}_{\infty}$. Based on the boundedness of the closed-loop signals, we can conclude that $\dot{u}_{d}(t) \in \mathcal{L}_{\infty}$; hence, we can utilize (105) and (108) to show that $\tau(t) \in \mathcal{L}_{\infty}$. Standard signal chasing arguments can now be used to show that all remaining signals remain bounded during closed-loop operation. Finally, the global asymptotic result given in (117) can now be directly obtained using the same procedure as given in the proof of Theorem 1.

\section{EXPERIMENTAL VERIFICATION}

\section{A. Experimental Configuration}

The adaptive tracking controller given by (16)-(20), (104), (105), and (107) was implemented on a modified K2A WMR manufactured by Cybermotion, Inc. The robot modifications include: 1) the replacement of the pulse-width modulated amplifiers with a dual channel Techron linear amplifier; 2) the re- placement of all existing computational hardware/software with a Pentium $133 \mathrm{MHz}$ PC; and 3) the replacement of the battery bank with an external power supply. Permanent magnet DC motors provide steering and drive actuation through a 106:1 and a 96: 1 gear coupling, respectively. A Pentium $133 \mathrm{MHz}$ PC operating under QNX (a real-time micro-kernel based operating system) hosts the control algorithm that was written in "C," and implemented using Qmotor 2.0 [9] (an in-house graphical user interface). Data acquisition and control implementation were performed at a frequency of $2.0 \mathrm{kHz}$ using the Quanser MultiQ I/O board. In order to measure the tracking error given in (7) we need to determine $x_{c}(t), y_{c}(t)$ and the orientation of the WMR. To this end, we obtained the positions of the steering and drive motors via Hewlett Packard (HEDS-9000) encoders with a resolution of $0.35 \mathrm{deg} / \mathrm{line}$, and then calculated the linear and angular velocity measurements via a filtered backward difference algorithm. Using the angular position measurement and the linear and angular velocity measurements, we then utilized the relationship given in (1) to determine $\dot{x}_{c}(t)$ and $\dot{y}_{c}(t)$. A trapezoidal integration routine was then applied to (1) to obtain $x_{c}(t)$ and $y_{c}(t)$. For simplicity, the electrical dynamics of the system were ignored. That is, we assumed that the computed torque is statically related to the voltage input of the permanent magnet DC motors by a constant.

\section{B. Experimental Results}

The dynamics for the modified K2A WMR are given as follows:

$$
\begin{aligned}
\frac{1}{r_{o}} & {\left[\begin{array}{cc}
1 & 0 \\
0 & \frac{L_{o}}{2}
\end{array}\right]\left[\begin{array}{l}
\tau_{1} \\
\tau_{2}
\end{array}\right] } \\
= & {\left[\begin{array}{cc}
m_{o} & 0 \\
0 & I_{o}
\end{array}\right]\left[\begin{array}{l}
\dot{v}_{1} \\
\dot{v}_{2}
\end{array}\right]+\left[\begin{array}{cc}
F_{s 1} & 0 \\
0 & F_{s 2}
\end{array}\right]\left[\begin{array}{l}
\operatorname{sgn}\left(v_{1}\right) \\
\operatorname{sgn}\left(v_{2}\right)
\end{array}\right] } \\
& +\left[\begin{array}{cc}
F_{d 1} & 0 \\
0 & F_{d 2}
\end{array}\right]\left[\begin{array}{l}
v_{1} \\
v_{2}
\end{array}\right]
\end{aligned}
$$

where

$$
\begin{array}{ll}
m_{o}=165 \mathrm{~kg} & \text { mass of the robot; } \\
I_{o}=4.643 \mathrm{~kg} \cdot \mathrm{m}^{2} & \text { inertia of the robot; } \\
r_{o}=0.010 \mathrm{~m} & \text { radius of the wheels; } \\
L_{o}=0.667 \mathrm{~m} & \text { length of the axis between the wheels; } \\
F_{s 1}, F_{s 2}, F_{d 1}, F_{d 2} & \text { dynamic and static friction elements. }
\end{array}
$$

The desired reference linear and angular velocity were selected as

$$
v_{r 1}=0.2(\mathrm{~m} / \mathrm{s}) \quad v_{r 2}=0.4 \sin (0.5 t)(\mathrm{rad} / \mathrm{s})
$$

respectively, (see Fig. 1 for the resulting reference time-varying Cartesian position and orientation).

The Cartesian positions and the orientation were initialized to zero, and the auxiliary signal $z_{d}(t)$ was initialized as follows:

$$
z_{d}(0)=\left[\begin{array}{ll}
0.01 & 0.01
\end{array}\right]^{T}
$$

The feedback gains were adjusted to reduce the position/orientation tracking error with the adaptation gains set to zero and all of the initial adaptive estimates set to zero. After some tuning, we noted that the position/orientation tracking error response could not be significantly improved by further adjustments of 


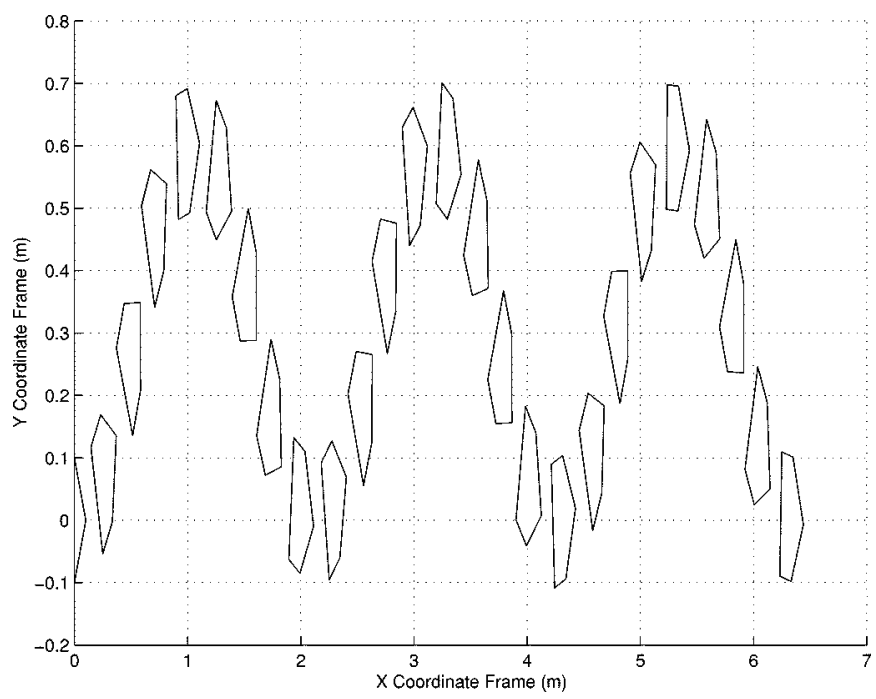

Fig. 1. Desired Cartesian position and orientation trajectory.

the feedback gains. We then adjusted the adaptation gains to allow the parameter estimation to reduce the position/orientation tracking error. After the tuning process was completed, the final adaptation and feedback gain values were recorded as shown below

$$
\begin{aligned}
k_{1} & =57, \quad k_{2}=2, \quad k_{3}=37 \\
K_{a} & =\left[\begin{array}{cc}
40 & 0 \\
0 & 1250
\end{array}\right] \\
\Gamma & =\operatorname{diag}\{30,0.05,300,50,300,10\} .
\end{aligned}
$$

The position/orientation tracking error of the COM of the WMR, the adaptive estimates, and the associated control torque inputs are shown in Figs. 2, 3, 4, and 5, respectively. (Note the control torque inputs plotted in Fig. 5 represent the torques applied after the gearing mechanism.) Based on Figs. 2 and 3 , it is clear that the steady-state position/orientation tracking error is bounded as follows:

$$
|\tilde{x}|<0.10 \mathrm{~cm} \quad|\tilde{y}|<0.22 \mathrm{~cm} \quad|\tilde{\theta}|<1.13 \mathrm{deg} .
$$

\section{CONCLUSION}

In this paper, we have presented a differentiable, kinematic control law for mobile robots. The proposed kinematic controller is novel in that 1) global exponential tracking was obtained provided certain PE conditions on the reference trajectory are satisfied and 2) a scheme was developed which solves the global exponential tracking problem and the global asymptotic regulation problem (provided a simple modification to the controller is made). Furthermore, the proposed kinematic controller is fundamentally different from the controller presented in [11] in the respect that 1) the proposed kinematic control structure does not require high gain feedback (i.e., we have eliminated the need to divide by a signal that exponentially approaches an arbitrarily small constant) and 2) we do not force the oscillator to track an exponentially decaying signal; hence, different analysis techniques are required. To illustrate that the proposed kinematic controller can be easily extended to include uncertain dy-

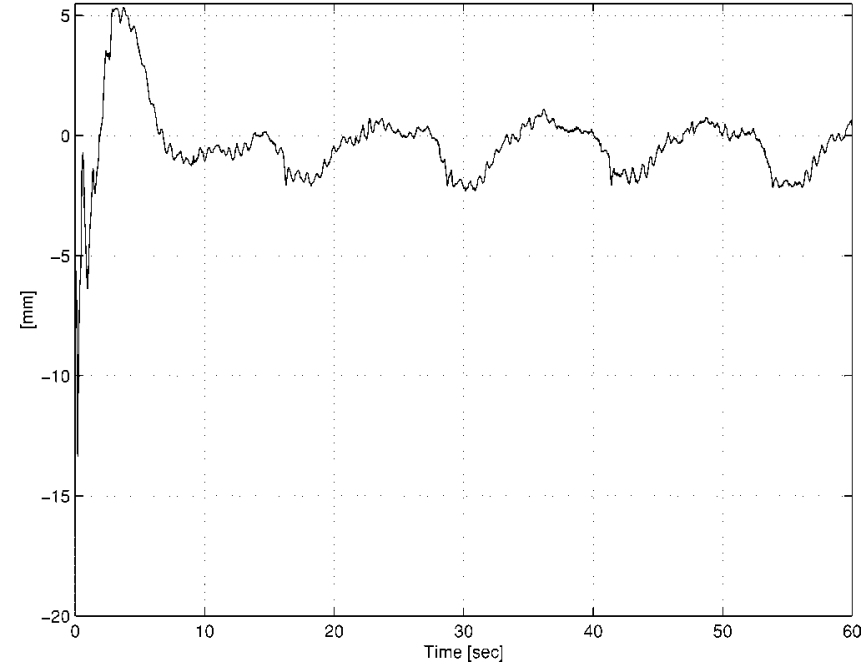

(a)

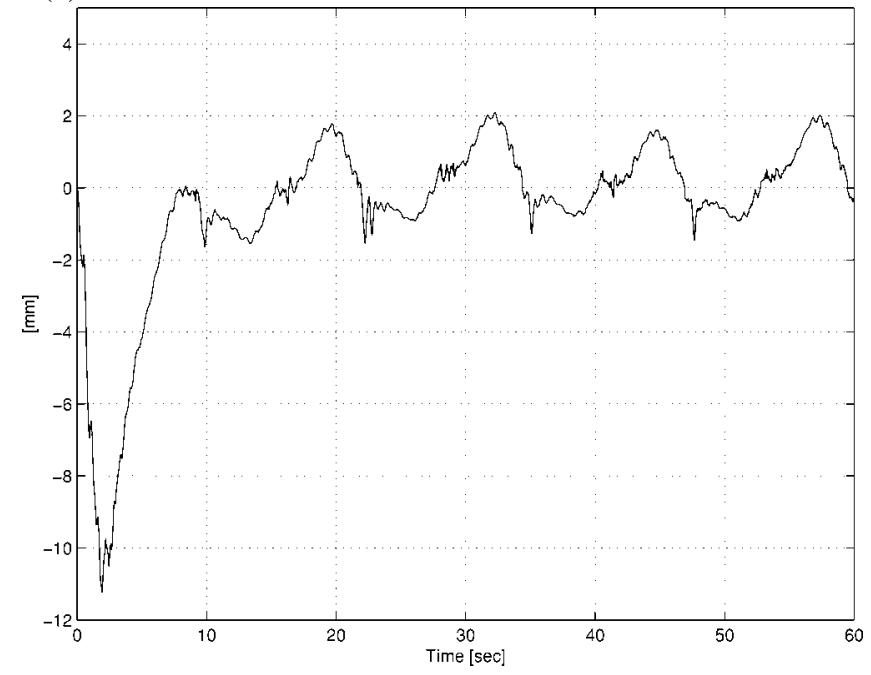

(b)

Fig. 2. Position tracking error: (a) $\tilde{x}$ and (b) $\tilde{y}$.

namic effects, we developed an adaptive controller that fosters global asymptotic tracking despite parametric uncertainty in the mobile robot dynamic model. It should also be noted that in addition to the WMR problem, the kinematic portion of the proposed controller can be applied to other nonholonomic systems (see [4] for examples). In addition, since the desired trajectory can be generated on-line (e.g., the desired trajectory could be calculated based on sonar data, vision-based data, etc.), the proposed controller could be used in many applications (e.g., obstacle avoidance, exploration of uncertain environments, etc.).

Experimental verification has been provided via a modified K2A WMR manufactured by Cybermotion, Inc. to illustrate the effectiveness of the proposed controller. In order to calculate the Cartesian position/orientation, we relied solely on position measurements from the steering and drive motor encoders; hence, we believe that if certain practical issues could be addressed (i.e., the motor encoders should be mounted before the WMR gearing mechanism, low resolution encoders $(0.35$ $\mathrm{deg} /$ line) should not be utilized, elimination of the numerical integration/differentiation error induced during the calculation of the Cartesian position/orientation, etc.), then the tracking error 


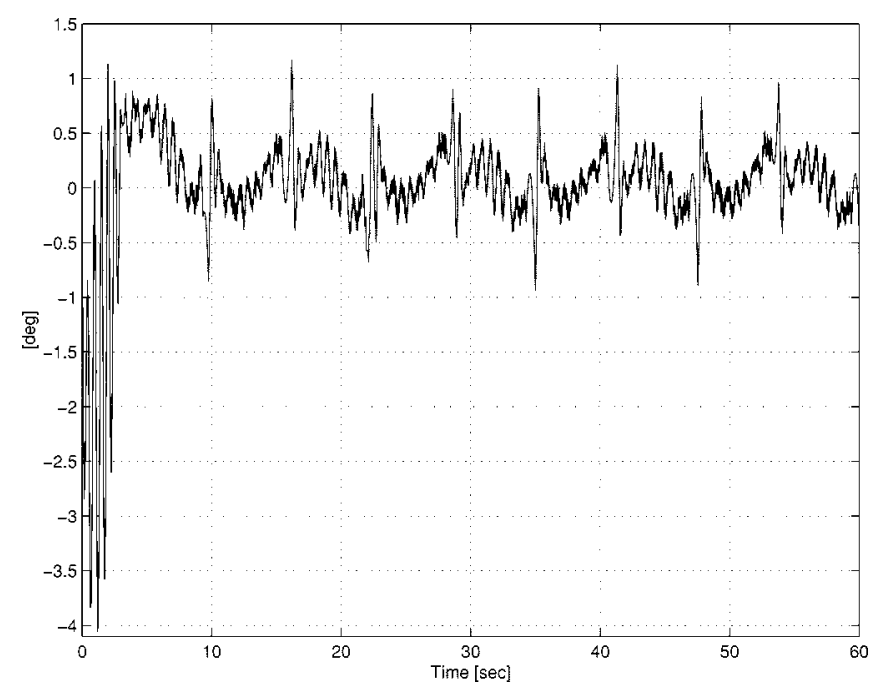

Fig. 3. Orientation tracking error $\tilde{\theta}$.
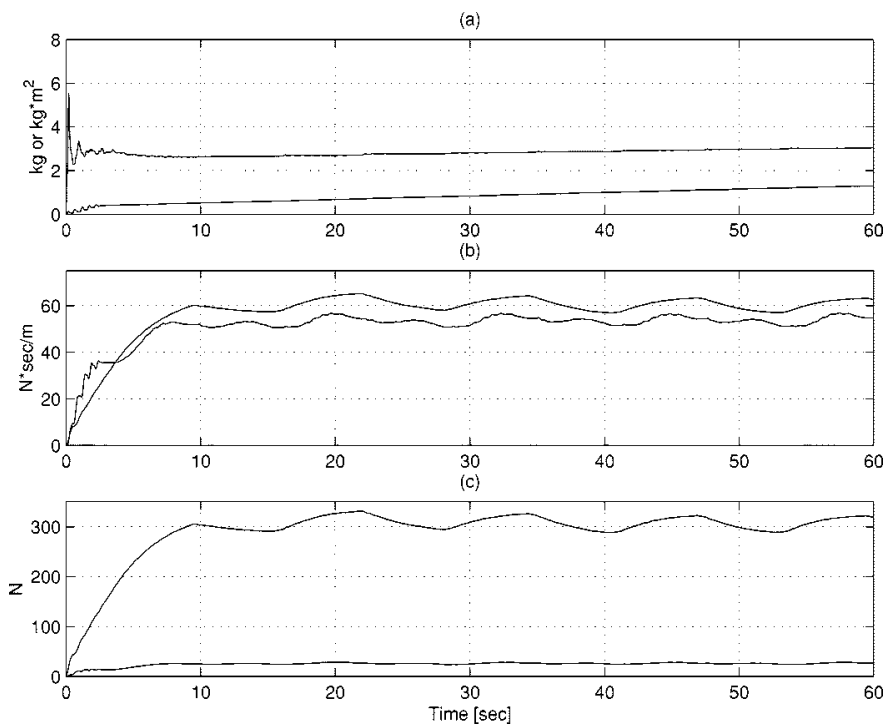

Fig. 4. Parameter estimates: (a) $m_{o}, I_{o}$, (b) $F_{d 1}, F_{d 2}$, and (c) $F_{s 1}, F_{s 2}$.

could be further decreased. Future research will address these issues by using visual feedback.

\section{APPENDIX}

In order to show that $\ddot{z}_{d}(t)$ is bounded we take the time derivative of (23) and then substitute for the time derivative of (20) and (10) to obtain the following expression:

$$
\begin{aligned}
\ddot{z}_{d}= & 2 k_{1}\left(w \dot{w}-z_{d}^{T} \dot{z}_{d}\right) z_{d}+\left(k_{1}\left(w^{2}-z_{d}^{T} z_{d}\right)-k_{2}\right) \dot{z}_{d} \\
& +J\left[\left(k_{1}+\Omega_{1}\right) \dot{w}+w \dot{\Omega}_{1}\right] z_{d}+J \Omega_{2} \dot{z}_{d} \\
& -\left[\frac{d}{d t}\left(I_{2}+2 w J\right)^{-1}\right] w A^{T}-\left(I_{2}+2 w J\right)^{-1} \dot{w} A^{T} \\
& -\left(I_{2}+2 w J\right)^{-1} w \\
& \cdot\left[\begin{array}{c}
\left.-2 \dot{v}_{r 1} \frac{\sin \left(z_{1}\right)}{z_{1}}-2 v_{r 1} \dot{z}_{1} \frac{z_{1} \cos \left(z_{1}\right)-\sin \left(z_{1}\right)}{z_{1}^{2}}\right] \\
2 \dot{v}_{r 2}
\end{array}\right] .
\end{aligned}
$$
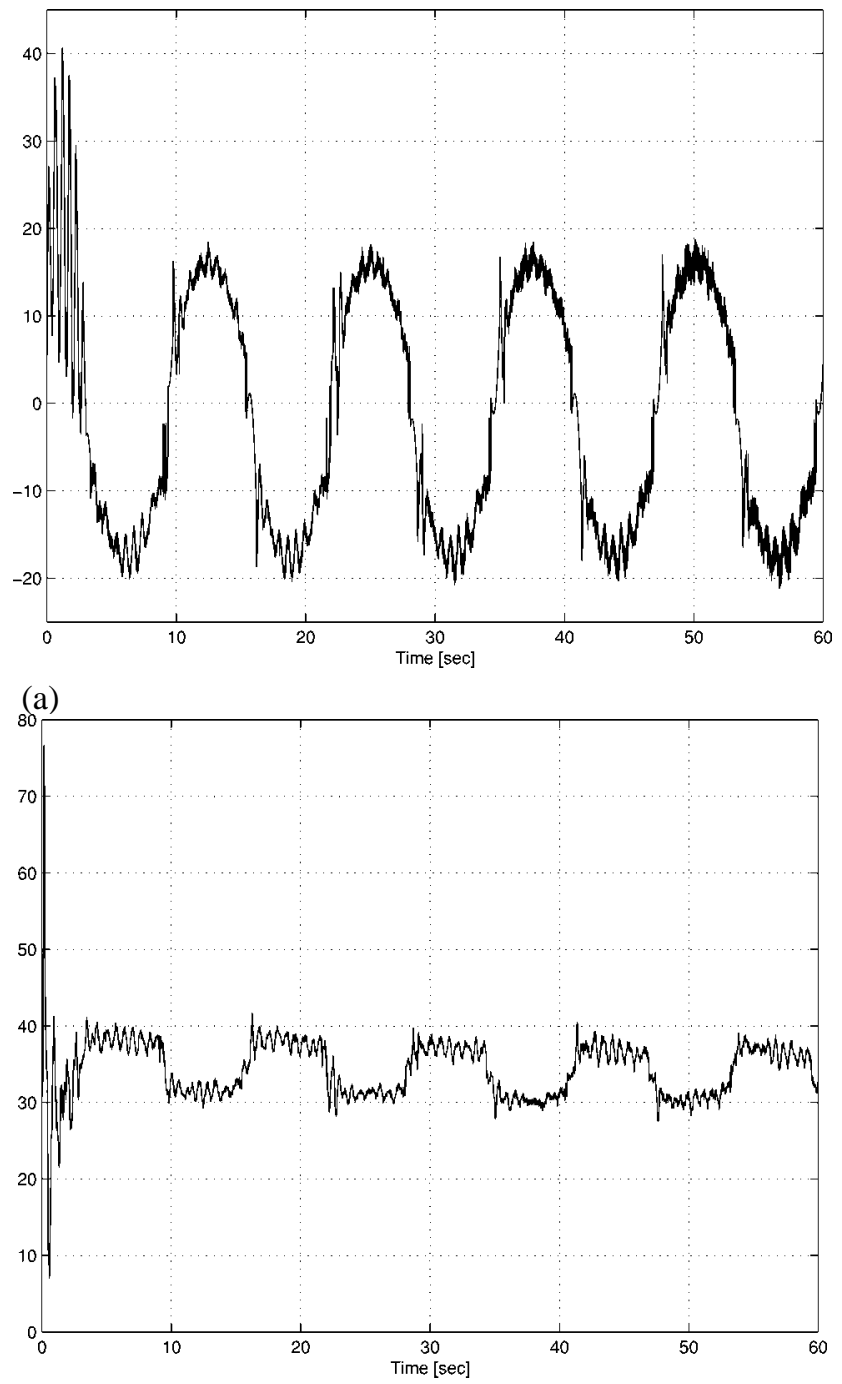

(b)

Fig. 5. Control torque input: (a) steering motor and (b) drive motor.

Then, substituting for the time derivative of (19) and grouping common terms yields

$$
\begin{aligned}
\ddot{z}_{d}= & 2 k_{1}\left(w \dot{w}-z_{d}^{T} \dot{z}_{d}\right) z_{d}+\left(k_{1}\left(w^{2}-z_{d}^{T} z_{d}\right)-k_{2}\right) \dot{z}_{d} \\
& +J \Omega_{2} \dot{z}_{d}-2 k_{1} w J z_{d}^{T} \dot{z}_{d} z_{d} \\
& +\left(\left(k_{1}+\Omega_{1}\right) J z_{d}+4 k_{1} w^{2} J z_{d}-\left(I_{2}+2 w J\right)^{-1} A^{T}\right) \dot{w} \\
& -\left(I_{2}+2 w J\right)^{-1} w \\
& \cdot\left[\begin{array}{cc}
-2 \dot{v}_{r 1} \frac{\sin \left(z_{1}\right)}{z_{1}}-2 v_{r 1} \dot{z}_{1} \frac{z_{1} \cos \left(z_{1}\right)-\sin \left(z_{1}\right)}{z_{1}^{2}} \\
2 \dot{v}_{r 2}
\end{array}\right] \\
& +\frac{2 w \dot{w}}{\left(1+4 w^{2}\right)^{2}}\left[\begin{array}{cc}
4 w & 4 w^{2}-1 \\
1-4 w^{2} & 4 w
\end{array}\right] A^{T} .
\end{aligned}
$$

After applying L'Hospital's rule to the terms contained in (128), which are given below

$$
\frac{\sin \left(z_{1}\right)}{z_{1}} \quad \frac{z_{1} \cos \left(z_{1}\right)-\sin \left(z_{1}\right)}{z_{1}^{2}}
$$


we note that

$$
\begin{aligned}
\lim _{z_{1} \rightarrow 0} \frac{\sin \left(z_{1}\right)}{z_{1}} & =1 \\
\lim _{z_{1} \rightarrow 0} \frac{z_{1} \cos \left(z_{1}\right)-\sin \left(z_{1}\right)}{z_{1}^{2}} & =0 .
\end{aligned}
$$

Finally, we can use (130) and the fact that $w(t), z_{d}(t), z(t)$, $\Omega_{1}(t), \Omega_{2}(t), v_{r}(t), \dot{z}_{d}(t), \dot{w}(t), \dot{z}(t), \dot{v}_{r}(t) \in \mathcal{L}_{\infty}$ to prove that $\ddot{z}_{d}(t) \in \mathcal{L}_{\infty}$.

\section{REFERENCES}

[1] L. E. Aguilar M, P. Soueres, M. Courdesses, and S. Fleury, "Robust pathfollowing control with exponential stability for mobile robots," in Proc. IEEE Int. Conf. Robot. Automat., 1998, pp. 3279-3284.

[2] B. D. O. Anderson, "Exponential stability of linear equations arising in adaptive identification," IEEE Trans. Automat. Contr., vol. 22, pp. 83-88, Feb. 1977.

[3] P. Antsaklis and A. Michel, Linear Systems. New York: McGraw-Hill, 1997.

[4] A. Bloch, M. Reyhanoglu, and N. McClamroch, "Control and stabilization of nonholonomic dynamic systems," IEEE Trans. Automat. Contr. vol. 37, Nov. 1992.

[5] R. Brockett, "Asymptotic stability and feedback stabilization," in Differential Geometric Control Theory, R. Brockett, R. Millman, and H. Sussmann, Eds. Boston, MA: Birkhauser, 1983.

[6] C. Canudas de Wit and O. Sordalen, "Exponential stabilization of mobile robots with nonholonomic constraints," IEEE Trans. Automat. Contr. vol. 37, pp. 1791-1797, Nov. 1992.

[7] C. Canudas de Wit, K. Khennouf, C. Samson, and O. J. Sordalen, "Nonlinear control for mobile robots," in Recent Trends in Mobile Robots, Y. Zheng, Ed. River Edge, NJ: World Scientific, 1993.

[8] J. Coron and J. Pomet, "A remark on the design of time-varying stabilizing feedback laws for controllable systems without drift," in Proc. IFAC Symp. Nonlinear Control Systems Design (NOLCOS), Bordeaux, France, June 1992, pp. 413-417.

[9] N. Costescu, D. M. Dawson, and M. Loffler, "Qmotor 2.0-A PC based real-time multitasking graphical control environment," IEEE Contr. Syst. Mag., pp. 68-71, June 1999

[10] D. Dawson, J. Hu, and P. Vedagharba, "An adaptive control for a class of induction motor systems," in Proc. IEEE Conf. Decision and Control, New Orleans, LA, Dec. 1995, pp. 1567-1572.

[11] W. E. Dixon, D. M. Dawson, E. Zergeroglu, and F. Zhang, "Robust tracking control of a mobile robot system," in Proc. IEEE Conf. Control Applications, Kohala Coast, HI, Aug. 22-26, 1999, pp. 1015-1020.

[12] W. Dong and W. Huo, "Adaptive stabilization of dynamic nonholonomic chained systems with uncertainty," in Proc. 36th IEEE Conf. Decision and Control, Dec. 1997, pp. 2362-2367.

[13] G. Escobar, R. Ortega, and M. Reyhanoglu, "Regulation and tracking of the nonholonomic double integrator: A field-oriented control approach," Automatica, vol. 34, no. 1, pp. 125-131, 1998.

[14] J. Godhavn and O. Egel, "A Lyapunov approach to exponential stabilization of nonholonomic systems in power form," IEEE Trans. Automat. Contr., vol. 42, pp. 1028-1032, July 1997.

[15] P. A. Ioannou and J. Sun, Robust Adaptive Control. Englewood Cliffs, NJ: Prentice-Hall, Inc., 1995.

[16] Z. Jiang and H. Nijmeijer, "Tracking control of mobile robots: A case study in backstepping," Automatica, vol. 33, no. 7, pp. 1393-1399, 1997.

[17] _ "A recursive technique for tracking control of nonholonomic systems in the chained form," IEEE Trans. Automat. Contr., vol. 44, pp. 265-279, Feb. 1999.

[18] Y. Kanayama, Y. Kimura, F. Miyazaki, and T. Noguchi, "A stable tracking control method for an autonomous mobile robot," in Proc. IEEE Int. Conf. Robot. Automat., 1990, pp. 384-389.

[19] H. K. Kahlil, Nonlinear Systems. Englewood Cliffs, NJ: Prentice-Hall, 1996.

[20] F. Lewis, C. Abdallah, and D. Dawson, Control of Robot Manipulators. New York: MacMillan, 1993.
[21] R. McCloskey and R. Murray, "Exponential stabilization of driftless nonlinear control systems using homogeneous feedback," IEEE Trans. Automat. Contr., vol. 42, pp. 614-628, May 1997.

[22] J. Pomet, "Explicit design of time-varying stabilizing control laws for a class of controllable systems without drift," Syst. Contr. Lett., vol. 18 , no. 2, pp. 147-158, 1992

[23] C. Samson, "Velocity and torque feedback control of a nonholonomic cart," in Proc. Int. Workshop Adaptive and Nonlinear Control: Issues in Robotics, Grenoble, France, 1990.

[24] - "Control of chained systems application to path following and time-varying point-stabilization of mobile robots," IEEE Trans. Automat. Contr., vol. 40, pp. 64-77, Jan. 1997.

[25] S. Sastry and M. Bodson, Adaptive Control: Stability, Convergence, and Robustness. Englewood Cliffs, NJ: Prentice-Hall, 1989.

[26] A. Teel, R. Murray, and C. Walsh, "Nonholonomic control systems: From steering to stabilization with sinusoids," Int. J. Control, vol. 62 , no. 4 , pp. 849-870, 1995 .

[27] G. Walsh, D. Tilbury, S. Sastry, R. Murray, and J. P. Laumond, "Stabilization of trajectories for systems with nonholonomic constraints," IEEE Trans. Automat. Contr., vol. 39, pp. 216-222, Jan. 1994.

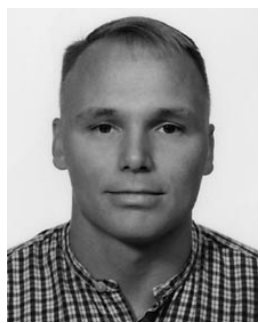

Warren Dixon was born in York, PA, in 1972 He received the B.S. degree in 1994 from the Department of Electrical and Computer Engineering of Clemson University, Clemson, SC., and the M.E. degree in 1997 from the Department of Electrical and Computer Engineering, University of South Carolina, Columbia. He is currently pursuing the Ph.D. degree at Clemson University.

As a student in the Robotics and Mechatronics group at Clemson University, his research is focused on nonlinear based robust and adaptive control techniques with application to electromechanical systems including wheeled mobile robots, twin rotor helicopters, surface vessels, and robot manipulators.

Darren M. Dawson (S'89-M'90-SM'94) was born in 1962, in Macon, GA. He received the A.S. degree in mathematics from Macon Junior College in 1982 and the B.S. and Ph.D. degrees in electrical engineering from the Georgia Institute of Technology, Atlanta, in 1984 and 1990, respectively

From 1985 to 1987, he was a Control Engineer at Westinghouse. While pursuing the Ph.D. degree at Georgia Institute of Technology, he also served as a Research/Teaching Assistant. In July 1990, he joined the Electrical and Computer Engineering Department and the Center for Advanced Manufacturing (CAM) at Clemson University, Clemson, SC, where he currently holds the position of Professor. Under the CAM director's supervision, he currently leads the Robotics and Manufacturing Automation Laboratory which is jointly operated by the Electrical and Mechanical Engineering departments. His main research interests are in the fields of nonlinear based robust, adaptive, and learning control with application to electromechanical systems including robot manipulators, motor drives, magnetic bearings, flexible cables, flexible beams, and high-speed transport systems.

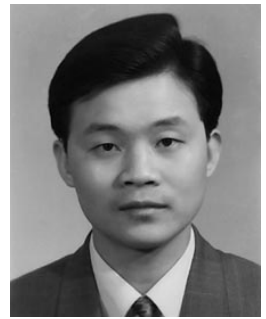

Fumin Zhang was born in Hancheng City, China, in 1970. He received the B.S. and M.S. degrees from the Department of Computer Science and Technology, Tsinghua University, Beijing, China, in 1993 and 1996, respectively, and the Ph.D. degree in computer engineering from the Department of Electrical Engineering and Computer Engineering, Clemson University, Clemson, SC, in May 1999. As a student in the Robotics and Mechatronics group at Clemson University, his research focused on nonlinear control techniques for mechatronic systems, boundary control of distributed parameter systems, and real-time software systems.

Upon completion of his education at Clemson University, he accepted the position of a Development Engineer at Seagate Technology, Inc., Bloomington, $\mathrm{MN}$. 


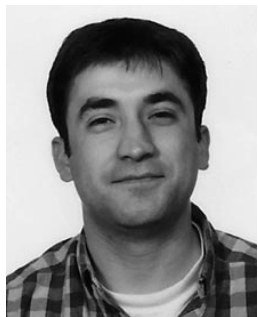

Erkan Zergeroglu was born in 1970, in Artvin-Yusufeli, Turkey. He received the B.S. degree in electrical engineering from Hacettepe University, Turkey, in 1992 and the M.S. degree from the Middle East Technical University, Turkey, in 1996. He is currently pursuin the Ph.D. degree at the Robotics and Mechatronics Laboratory, Clemson University, Clemson, SC. His research interests are nonlinear control of electromechanical systems with parametric uncertainty, and applied robotics. 